\title{
Operando Imaging of Ion Migration in Metal Halide Perovskites
}

Yongtao Liu ${ }^{1}$, Anton Ievlev $^{2}$, Liam Collins ${ }^{2}$, Nikolay Borodinov², Sergei Kalinin ${ }^{2}$ and Olga Ovchinnikova $^{2}$

${ }^{1}$ The University of Tennessee, Knoxville, Knoxville, Tennessee, United States, ${ }^{2}$ Oak Ridge National Laboratory, Oak Ridge, Tennessee, United States

Metal halide perovskites (MHPs) have attracted tremendous interest due to the rapidly growing photovoltaic efficiency [1]. Meanwhile, this class of materials is characterized by the presence of the ion migration and ion redistribution in operando conditions [2], which play important roles in the functionality of MHPs [3-10]. Therefore, the development of MHPs-based optoelectronic devices necessitates a precise understanding concerning the interplay between ionic dynamics and the photoelectric processes. However, up to date, most works probe the ion migration in MHPs indirectly or in a static regime, direct observation of ion migration in operando condition is missing.

In this work, using a newly developed time-resolved time-of-flight secondary ion mass spectrometry (trToF-SIMS, shown in Figure 1a) we show a direct observation of real-time ion migration (including methylammonium $\left(\mathrm{CH}_{3} \mathrm{NH}_{3}{ }^{+}\right)$and halides $\left(\mathrm{I}^{-}\right.$and $\left.\mathrm{Br}^{-}\right)$) induced by light illumination and electric bias in methylammonium lead iodide $\left(\mathrm{CH}_{3} \mathrm{NH}_{3} \mathrm{PbI}_{3}\right)$ and methylammonium lead bromide $\left(\mathrm{CH}_{3} \mathrm{NH}_{3} \mathrm{PbBr}_{3}\right)$. We use a lateral device with two Au electrodes on MHP films (shown in Figure 1b) to perform in-situ operando studies. We reveal that photoexcitation can induce a large redistribution of $\mathrm{CH}_{3} \mathrm{NH}_{3}{ }^{+}$in the lateral devices, where $\mathrm{CH}_{3} \mathrm{NH}_{3}{ }^{+}$is uniformly distributed between two Au electrodes (shown in Figure 2a) under light illumination but concentrates at the center under dark (shown in Figure 2b). Although it was generally believed that light-illumination can enhance ion migration and induce chemical segregation, our direct observation by ToF-SIMS in Figure 2c-d shows that light-illumination suppresses the electric bias induced ion segregation in MHP. With the assistance of the machine learning analysis approach, we unveil the migration of not only the intrinsic ions (e.g. $\mathrm{CH}_{3} \mathrm{NH}_{3}{ }^{+}$and $\mathrm{I}^{-} / \mathrm{Br}^{-}$) in MHPs but also the contaminants and the degradation products, offering a full picture of ion migration in MHPs. By applying a bi-polar waveform bias, we show that the ion migration in $\mathrm{CH}_{3} \mathrm{NH}_{3} \mathrm{PbI}_{3}$ is hysteretic. In addition, the bias-driven ion migration can induce a remanent field in MHPs, which further results in a remanent current that also shows a hysteresis. We believe this remanent field induced by ion redistribution accounts for the currentvoltage hysteresis in the MHP-based solar cells. Moreover, combining with the time-resolved Kelvin probe force microscopy (tr-KPFM), we observed the photoinduced surface potential changes in MHPs are opposite to the ion dynamics. These pieces of knowledge were unknown previously, suggesting the importance of the direct observation of ion migration in MHPs using tr-ToF-SIMS [11]. 


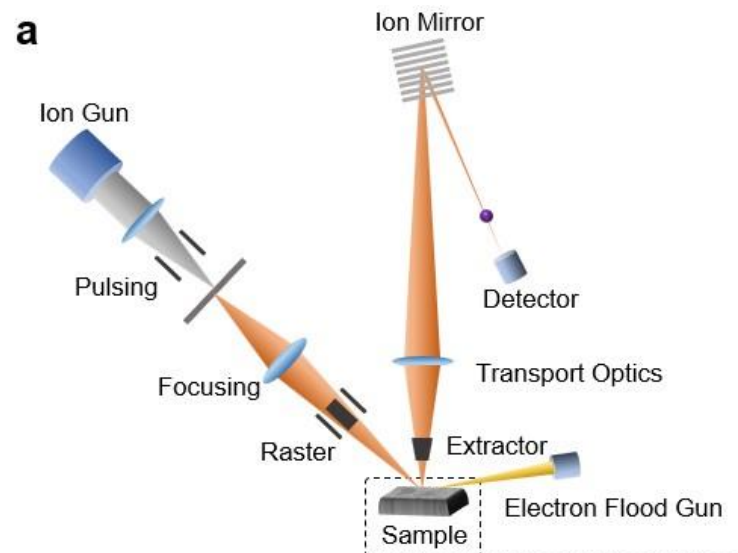

b

Figure 1. a, a scheme of the functional principle of ToF-SIMS; b, scheme of the lateral device $\mathrm{Au} / \mathrm{MHP} / \mathrm{Au}$ used in this study, where a scanning electron microscope (SEM) image of $\mathrm{CH} 3 \mathrm{NH} 3 \mathrm{PbI} 3$ was embedded.
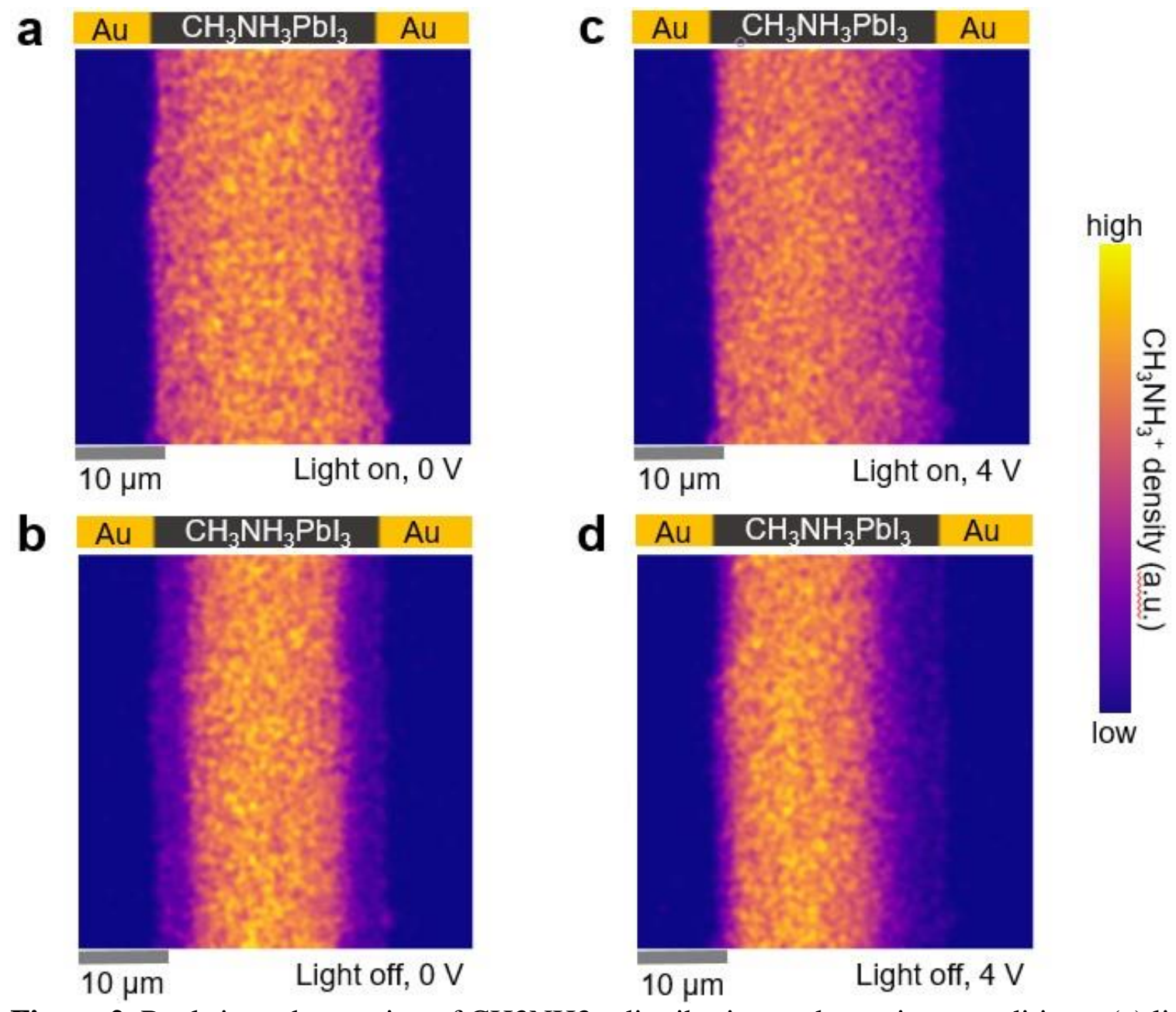

Figure 2. Real-time observation of $\mathrm{CH} 3 \mathrm{NH} 3+$ distribution under various conditions: (a) light on and $0 \mathrm{~V}$, (b) light off and $0 \mathrm{~V}$, (c) light on and $4 \mathrm{~V}$ poling, (d) light off and $4 \mathrm{~V}$ poling. 


\section{References}

[1] HJ Snaith, Nature materials 17 (2018), p. 372.

[2] Y Yuan and J Huang, Accounts of chemical research 49 (2016), p. 286.

[3] Y Liu et al., Nature materials 17 (2018), p. 1013.

[4] Y Liu et al., Nature materials 18 (2019), p. 1051.

[5] Y Liu et al., Applied Physics Letters 113 (2018), p. 072102.

[6] Y Liu et al., Advanced Optical Materials 7 (2019), p. 1901451.

[7] Y Liu et al., Advanced Electronic Materials, (2020), p. 1901235.

[8] Y Liu et al., APL Materials 8 (2020), p. 011106.

[9] Y Liu et al., MRS Advances 4 (2019), p. 2817.

[10] L Collins et al., Nanotechnology 29 (2018), p. 445703.

[11] Research was conducted at Oak Ridge National Laboratory's Center for Nanophase Materials Sciences (CNMS), which is a U.S. Department of Energy Office of Science User Facility. 